\title{
The Clinical Characteristics and Emergent Treatments of Patients with Anaphylactic Shock
}

\author{
Guoxiang Zhang*
}

Huantai Branch of Qilu Hospital of Shandong University, Zibo, Shandong 256400, China

\begin{abstract}
Objective: To analyze the clinical characteristics of patients with anaphylactic shock, and discuss the appropriate emergent treatments. Methods: 18 cases of anaphylactic shock patients received emergent treatments in our hospital from October 2012 to October 2014 were randomly selected. Retrospective analysis was made on the basic information such as clinical characteristics, emergency treatments, etc. Results: Through retrospective analysis, it was found out that the drug-induced anaphylactic shock accounted for $88.89 \%$, biotoxins-induced anaphylactic shock accounted for $5.55 \%$, and blood transfusion-induced anaphylactic shock accounted for $5.55 \%$. The main clinical characteristics of anaphylactic shock are the illness occurred suddenly, developed fast, hard to predict, without indication, and can cause death, etc. The recovery rate reached $94.44 \%$ after emergent treatments, but there was only 1 case of anaphylactic shock patients who died because of not getting timely treatment. Conclusion: Understanding the clinical characteristics of anaphylactic shock, timely and effective treatment measures are the keys of successful treatment.
\end{abstract}

\author{
KEYWORDS \\ Anaphylactic shock \\ Clinical characteristic \\ Emergent treatment
}

\section{Introduction}

Anaphylactic shock is a relatively rare systemic anaphylaxis reaction. The sensitized body is invaded by external antigen substances, the immune mechanism lead to strongly multiple organs involvement syndrome in a short time and if not been treated in time will cause death [1]. Clinical reports [2] think the anaphylactic shock have complex causes, has the characteristics of hard to predict and fast onset, and contributed to great threat to anaphylactic shock patient's life health. It is one of the important prevention

Copyright @ 2016 Guoxiang Zhang

doi: 10.18686/aem.v5i3.11

Received: August 3, 2016; Accepted: September 5, 2016; Published online: September 14, 2016

This is an open-access article distributed under the terms of the Creative Commons Attribution Unported License (http://creativecommons.org/ licenses/by-nc/4.0/), which permits unrestricted use, distribution, and reproduction in any medium, provided the original work is properly cited.

${ }^{\star}$ Corresponding author: Huantai Branch of Qilu Hospital of Shandong University, Zibo, Shandong 256400, China. E-mail: 003zhuanx@sina. com diseases of clinical emergency treatment. This research made a retrospectively analysis on the clinical data and treatment of 24 anaphylactic shock patients in our hospital and provided the reference for clinical treatment of this kind emergency treatment diseases.

\section{Data and Methods \\ 2.1. General Data}

18 cases of anaphylactic shock patients received treatment in our hospital from October 2012 to October 2014 were randomly selected, with 10 males and 8 females. The ages were between 14-64 years old, the average age was(30.6 \pm 5.4 years old. The clinical investigation data showed all the selected anaphylactic shock patients had no history of allergies and they had anaphylactic shock within 3-42 min after they had contact with allergens, average of (7.6 \pm 1.4) min. There were 3 cases occurred within 5 minutes, 12 cases occurred between 5-30 min and 1 case occurred after more than $30 \mathrm{~min}$. The basic data of anaphylactic shock patients did not affect study. 
Table 1. Main System Distributions of Allergic Shock Clinical Manifestations.

\begin{tabular}{|c|c|}
\hline Involved System & Clinical Manifestation \\
\hline Breathing System & $\begin{array}{l}\text { Prang asthma, chest congestion, breathing difficulties associated with dying, lung wheezing sound moist } \\
\text { crackles, laryngeal edema, nasal obstruction, no breathing. } \\
\text { Pale face, lower blood pressure, chest tightness, heart palpitations, accelerated heart rate, wet and cool body, }\end{array}$ \\
\hline Cardiovascular System & $\begin{array}{l}\text { blue jaundice of lip or acrocyanosis, pulse is thin or can't be tested, cardiac arrest, weak or disappeared heart } \\
\text { sound. }\end{array}$ \\
\hline Nervous System & $\begin{array}{l}\text { Restlessness, head bilges, lightheadedness, chills, limb numbness, cold sweat, loss of consciousness, } \\
\text { convulsions. }\end{array}$ \\
\hline Gastroenteric System & Nausea, vomiting, abdominal distension, abdominal pain, diarrhea, gatism. \\
\hline Urinary System & Facial edema, urinary incontinence. \\
\hline Skin and Appendages & Itching, skin flush, urticarial. \\
\hline Eyes & Conjunctival congestion, giddiness, blurred vision, blacked out. \\
\hline Other & Hearing impairment, tinnitus. \\
\hline
\end{tabular}

\subsection{Research Methods}

Retrospective analysis was made on the basic information such as clinical characteristics, performance and emergency treatment and others of 18 cases of anaphylactic shock patients.

\subsection{Emergency Treatment}

18 cases of anaphylactic shock patients were treated by the following emergency measures: (1) Take the anaphylactic shock patients away from the allergen immediately. For the patients caused by blood transfusion, stopped transfusion immediately and sealed up for inspection. For the patients caused by drug, stopped the drug immediately and closely observed the vital signs of anaphylactic shock patients. (2) Gave the anti-shock treatment, selected intramuscular injection of $0.5-1 \mathrm{mg}$ epinephrine hydrochloride $(0.1 \%)$ according to the tolerance of anaphylactic shock patients. The dosage of children is around $0.025 \mathrm{mg} / \mathrm{kg}$, then intravenous drip of dopamine $(5-10 \mu \mathrm{g} / \mathrm{kg} / \mathrm{min})$ was gave to maintain according to the blood pressure of patients, $1000-2000 \mathrm{~mL}(1 / 3$ colloidal solution $+2 / 3$ crystalloid solution) was administrated fast within 120 min. (3) Gave the anti-allergy treatment, gave high-dose of dexamethasone or hydrocortisone immediately, at the same time, use antihistamine drugs diphenhydramine or promethazine hydrochloride according to the situation of anaphylactic shock patients, and appropriately gave vitamin $\mathrm{C}$ and calcium, etc. (4) The treatment of complications, those with severe laryngeal edema, prepared for tracheotomy, and gave cricothyroid membrane puncture, those with bronchial asthma, gave spasmolysis and asthma treatment. (5) Besides that, treated the anaphylactic shock patients with the following methods: keep warm, oxygen intake, smooth respiratory tract and symptomatic treatment, etc. In the process of the above treatment, a closed observation should be made on anaphylactic shock patient's blood pressure, heart rate, pulse, oxygen saturation, consciousness, etc. and give the laboratory test if necessary.

\section{Results \\ 3.1. Clinical Manifestations}

Through the clinical manifestation of the 18 cases of anaphylactic shock patients, it was found that 16 cases of anaphylactic shock patients were indifferent, 14 cases of anaphylactic shock patients had the symptoms of nausea, dizziness, palpitation, chest tightness, etc; 4 cases had the symptoms of itching and urticarial, etc; 18 cases of anaphylactic shock patients had arterial pressure not exceeded $70 \mathrm{mmHg}$, all of them had sallow breathing; 2 cases had the symptoms of difficult breathing. The clinical manifestations' main system distributions are shown in Table 1.

\subsection{Allergen}

Through the retrospectively analysis, it was found that the drug-induced anaphylactic shock accounted for $88.89 \%$. Among them, antimicrobials accounted for 7 cases, mostly penicillin and cephalosporin drugs; Chinese patent drug accounted for 6 cases, mostly Shuanghuanglian and compound salvia miltiorrhiza drugs; other drugs accounted for 3 cases, biotoxins accounted for 5.55\%; bee sting accounted for 1 case, blood transfusion accounted for 5.55\%; and blood products accounted for 1 case.

\subsection{Emergency Treatment Results}

After the symptomatic treatment of 18 cases of anaphylactic shock patients, 17 cases of anaphylactic shock patients are recovered. The recovery rate reached $94.44 \%$ and 1 case of anaphylactic shock patients were died due to not having a timely treatment.

\section{Discussion}

\subsection{Anaphylactic Shock}

Anaphylactic shock is a kind of severe clinical emergency treatment diseases. The disease can cause damage to solid organs in a short time and trigger systemic allergic reaction. It will cause death in a serious condition, has serious impact on allergic shock patients' life. Most of the 
clinical studies have shown that the cause of the disease is complex, mainly caused by external sensitization antigen that invading the body. The main antigenic substances include polysaccharide, medicine, biological toxin, blood products, etc. The anaphylactic shock patients' allergic threshold is very low, therefore, we should avoid drug abuse during the emergency treatment, in order to avoid other inflammation reaction [3].

\subsection{Analysis of Clinical Characteristics}

The main clinical manifestations of anaphylactic shock include haziness of spiritual mind, nausea, dizziness, palpitation, chest tightness, low blood pressure, itching, hives, breathing difficulties, etc. The organ system that mainly involved include respiratory system, cardiovascular system, nervous system, gastrointestinal system, urinary system, skin and accessories, etc. In this study, $94.44 \%$ anaphylactic shock patients got shock within 30 minutes, the results indicated that the disease has the clinical characteristics of rapid and critical. The analysis showed that among the 18 anaphylactic shock patients, 6 cases of anaphylactic shock patients did not show any sign when shock occurred, which showed most of the anaphylactic shock had no clinical signs. What is more, anaphylactic shock has the clinical characteristics of hard to predict, complex allergen and causing death, etc. Therefore, mastering the clinical characteristics of the disease has very important meaning for the disease emergent treatment [4].

\subsection{Analysis of Emergency Treatment}

The measures for anaphylactic shock patients must be took in time. The emergent treatments such as keeping away from the allergen, anti-shock therapy, anti-allergic treatment and prevention of complications were took for allergic shock patients. Keeping away from the allergen is to cut off the source of the reaction, and provide a basis for follow-up work of emergency treatment. Adrenaline used for antishock treatment has the utility of fast diastolic bronchospasm and shrinking small peripheral blood vessels. It also can fight with abnormal reaction media release, regulate and control many links of immune response. Therefore, the drug is the first selection medicaments of anti-shock [5]. Anti-allergic treatment mainly used the dexamethasone or hydrocortisone. Dexamethasone has anti-inflammatory, antirheumatism, anti-allergic and immune suppression effect. Hydrocortisone is a natural glucocorticoid, which the antiinflammatory effect is remarkable. Diphenhydramine is a kind of antihistamines drug. It has the effect of restraining central nervous and has certain relief for the inflammation caused by anaphylactic shock. Vitamin $\mathrm{C}$ and calcium supplements can replenish nutrition and improve allergic shock patients' immune function. Allergic shock patients will be followed by symptoms such as laryngeal edema, bronchial asthma, etc. whether these complications are treated correctly will affect the rescue work, and critical situation will lead to death, while symptomatic treatment can effectively relieve such complications. In the process of emergency treatment, pay close attention to the vital signs of anaphylactic shock patients, which can provide certain references for emergent treatments [6]. In this research, after symptomatic treatment measures of the 18 cases of anaphylactic shock patients, 17 cases of patients were recovered. The recovery rate reached $94.44 \% .1$ case of anaphylactic shock patients died because of not having timely treatment. This study achieved satisfactory results through timely and effective emergency treatment, it also illustrated that the effective diagnosis and treatment is the important guarantee of successful cure of allergic shock patients.

\subsection{Conclusion}

By analyzing the clinical data of 18 patients with anaphylactic shock, it is believed that mastering the clinical characteristics of anaphylactic shocks have an important diagnosis and guidance significance for the emergency treatment. Emergency physicians and nurses should understand and update the knowledge timely. In response to save this kind of urgent disease, we should find and judge promptly, choose the right measures according to the situation of anaphylactic shock patients. At the same time, quickly carry out the rescue work, to ensure the life and health of anaphylactic shock patients.

\section{References}

1. Xu QH. 21 Cases of Anaphylactic Shock Patients' Clinical Characteristics and First Aid Experience. Chinese Critical Disease Emergency Medicine. 2009;21(5):310.

2. Zhao HM, Zheng WJ, Liu JH, et al. Anaphylactic Shock's Clinical Characteristics and First Aid Experience with Analysis of 24 cases. New medicine. 2008;33(7)6:470-471.

3. Jia ZL, Li ZK. Treatment Experience on 12 Cases of Druginduced Anaphylactic Shock. Chinese Modern Clinical Medical Journal. 2007;5(6):527.

4. Qi KL, Chen ZC, Liu YX, et al. Clinical Analysis of 33 Cases of Allergic Shock. Lingnan Journal of Emergency Medicine, 2010;15(2):120-121.

5. Shi YL, He XQ. Drug-induced Anaphylactic Shock's Emergency Treatment. Health Must Read Magazine (Late Ten-Day Publication). 2010(10):260.

6. Paul-Georg G, Jörg K, Dieter H, Wolfram S, Dietrich H. Intratracheally applied rSP-C surfactant exhibits no Anaphylactic shock reactions in a guinea pig model of acute lung hypersensitivity. Experimental and Toxicologic Pathology. 1999:516. 